FERMILAB-PUB-89/249-A

November 1989

\title{
THE DYNAMICS OF DOMAIN WALLS AND STRINGS
}

\author{
Ruth Gregory \\ David Haws \\ N.A.S.A./ Fermilab Astrophysics Center, Fermi National Accelerator Laboratory \\ P.O.Box 500, Batavia, IL 60510, U.S.A. \\ and \\ David Garfinkle \\ Department of Physics, University of California, Santa Barbara, CA 93106.
}

\begin{abstract}
The leading order finite-width corrections to the equation of motion describing the motion of a domain wall are derived. The régime in which this equation of motion is invalid is discussed. Spherically and cylindrically symmetric solutions to this equation of motion are found. We also clarify a misconception that has arisen in recent years regarding the rigidity (or otherwise) of cosmic strings.
\end{abstract}


There has recently been renewed interest in domain walls as possible sources of large scale structure in the universe ${ }^{1}$. With a few exceptions, most of the work done on the motion of walls has used the zero thickness approximation. The numerical studies of Widrow ${ }^{2}$ and Press, Ryden and Spergel ${ }^{3}$ have given much insight into the dynamics and evolution of finite thickness walls; however, it would be desirable to develop an analytic description of domain wall evolution which would preferably be model independent. In other words, we would like to obtain an approximate equation for a wall surface without having to calculate the detailed dynamics of the scalar field.

We start by deriving the equations of motion for an infinitely thin domain wall (which turns out to be a generalization of the Nambu equation of motion for strings). Then we derive the leading order corrections to this equation for walls of finite thickness due to the extrinsic curvature of the wall and the wall's self gravity. We do this by using an expansion scheme for the field theoretic equations of motion. For the non-gravitating case we check that the results obtained using the less satisfactory "effective action" method are the same. Next we comment on a misconception that has arisen in recent years regarding the rigidity of cosmic strings ${ }^{4}$. Returning to walls, we proceed by discussing the relative importance of the gravitational and field theoretic correction terms, and under which circumstances each are relevant. We then apply our equations to a few simple cases to illustrate the rigidifying (or otherwise) effect of the correction. We compare our results with those of Widrow, and discuss the breakdown of our approximation.

A domain wall can occur during a phase transition into a broken symmetry state where the vacuum manifold has two or more disconnected regions. The wall becomes the boundary between regions lying in different vacua. To model the essential features of a thick wall, we will consider a scalar field with Lagrangian

$$
L=-\frac{1}{2} \nabla_{a} \phi \nabla^{a} \phi-V(\phi)
$$

(we shall take the metric to be of the form $(-,+,+,+)$ ) and equation of motion

$$
\nabla_{a} \nabla^{a} \phi-\frac{\partial V(\phi)}{\partial \phi}=0
$$


which contain a $\phi \rightarrow-\phi$ symmetry. $V(\phi)$ is assumed to have two or more minima at non-zero values of $\phi$. Typically, $V(\phi)$ is taken to be a Sine-Gordon or $\lambda \phi^{4}$ potential. We will consider the case where $\langle\phi\rangle \rightarrow \pm\langle\phi\rangle_{0}$ on either side of the wall, and therefore define the wall surface, $\Sigma$, to be the three dimensional surface on which $\phi=0$. In the typical example of a $\lambda \phi^{4}$ wall in flat space, we have

$$
\begin{aligned}
V(\phi) & =\lambda\left(\phi^{2}-\eta^{2}\right)^{2} \\
\phi_{0} & =\eta \tanh (\sqrt{2 \lambda} \eta z) \\
T_{a b} & =-2 \lambda \eta^{4} \operatorname{sech}^{4}(\sqrt{2 \lambda} \eta z)\left(g_{a b}-\nabla_{a} z \nabla_{b} z\right) .
\end{aligned}
$$

In this case, we see that

$$
\begin{aligned}
& \epsilon=\frac{1}{\sqrt{2 \lambda} \eta} \\
& \sigma=\frac{4}{3} \sqrt{2 \lambda} \eta^{3}
\end{aligned}
$$

represent the thickness and energy per unit area of this wall respectively; these will be our expansion parameters for the wall equations. More generally, if $\eta$ represents the symmetry breaking scale, and $\lambda$ the self coupling of the field, then we expect the thickness of the wall always to be $O(1 / \sqrt{\lambda} \eta)$ and its energy per unit area $O\left(\sqrt{\lambda} \eta^{3}\right)$.

This is the solution for a flat domain wall in a flat spacetime, in order to model a more general domain wall, we need to assume that the thickness of the wall is much smaller than both the extrinsic curvature of the wall and the horizon length (which is of order $(G \sigma)^{-1}$ ). We may therefore split the field equations into their components orthogonal and tangential to $\Sigma$, the wall surface. This was done in ref. 5 using a Gauss Codazzi formalism, and so we will merely paraphrase the method here, and refer the reader to [5] for the details.

We let $n^{a}$ be a unit geodesic normal vector field to $\Sigma$, and let $z$ be the proper length along the integral curves of $n^{a}$. Each $z=$ const. surface then has unit normal $n_{a}$, intrinsic metric $h_{a b}$ and extrinsic curvature $K_{a b}$ defined by

$$
\begin{aligned}
h_{a b} & =g_{a b}-n_{a} n_{b} \\
K_{a b} & =h_{a}^{c} \nabla_{c} n_{b} .
\end{aligned}
$$


One can now use the Gauss Codazzi formalism to write the coupled Einstein-scalar equations in terms of $h_{a b}, K_{a b}$ and $\phi$ :

$$
\begin{gathered}
\mathcal{L}_{n} h_{a b}=2 K_{a b} \\
\mathcal{L}_{n} K_{a b}={ }^{(a)} R_{a b}+2 K_{a c} K_{b}^{c}-K K_{a b}-8 \pi G D_{a} \phi D_{b} \phi-8 \pi G V(\phi) h_{a b} \\
\mathcal{L}_{n} \mathcal{L}_{n} \phi+K \mathcal{L}_{n} \phi+D_{a} D^{a} \phi-\frac{\partial V(\phi)}{\partial \phi}=0
\end{gathered}
$$

where $D_{a}$ and ${ }^{(3)} R_{a b}$ are the derivative operator and Ricci tensor of the wall surface.

Now consider the new variables

$$
u=z / \epsilon \quad X=\phi / \eta
$$

with $\epsilon$ defined by (1), then $\mathcal{L}_{n}=\frac{1}{\epsilon} \frac{\partial}{\partial u}$ in this coordinate system. Moreover, since we can set $V(\phi)=\lambda \eta^{4} U(X)=\frac{3}{8} \epsilon^{-1} \sigma U(X)$, where $U(X)$ will now be $O(1)$, we may rewrite equations (3) as

$$
\begin{gathered}
\frac{\partial h_{a b}}{\partial u}=2 \epsilon K_{a b} \\
\frac{\partial K_{a b}}{\partial u}=-3 \pi G \sigma U(X) h_{a b}+\epsilon\left(^{(a)} R_{a b}+2 K_{a c} K_{b}^{c}-K K_{a b}\right)-6 \pi G \sigma \epsilon^{2} D_{a} X D_{b} X \\
\quad \frac{\partial^{2} X}{\partial u^{2}}-\frac{1}{2} \frac{\partial U(X)}{\partial X}+\epsilon K \frac{\partial X}{\partial u}+\epsilon^{2} D_{a} D^{a} X=0 .
\end{gathered}
$$

We now write our solutions in terms of a power series in $\epsilon$. Clearly the zeroth order solution to equation (4b) is $X=X_{0}(u)$, where $X_{0}(u)$ is the flat space planar wall solution ( $\tanh u$ for the $\lambda \phi^{4}$ wall). We also see that $h_{0 a b}$ is constant, and $K_{0 a b}$ is given by

$$
K_{o a b}=K_{0 a b}(0)-3 \pi G \sigma h_{0 a b} \int d u U(X)
$$

which is just a constant term plus an odd function of $u$.

Taking the difference of this last expression on either side of the wall yields the Israel equations for the exterior spacetime to the wall. On either side of the wall the Einstein 
constraint equation ${ }^{(8)} R+K_{a b} K^{a b}-K^{2}=0$ is satisfied. Taking the difference of the constraint equation on either side of the wall and using the Israel equation we find that $K_{0}(0)=0$. There is another way to derive this result using only the scalar equation (4b). Multiply equation (4b) by $\partial X / \partial u$ and integrate from $-\infty$ to $\infty$ using the boundary conditions on $X$ to obtain

$$
\int_{-\infty}^{\infty} d u\left[\left(X^{\prime}\right)^{2} K+\epsilon X^{\prime} D_{a} D^{a} X\right]=0
$$

Here a prime denotes derivative with respect to $u$. Now evaluating equation (5) to zeroth order in $\epsilon$ we find

$$
\begin{aligned}
\int_{-\infty}^{\infty} d u\left(X_{0}^{\prime}\right)^{2} K_{0} & =0 \\
\Rightarrow \quad K_{0}(0) & =0 .
\end{aligned}
$$

But this is just the wave equation for the wall surface. Thus, to zeroth order in wall thickness independent of the wall model, or any ansatz for the fields and even independent of gravity, the wall must obey a 'Nambu' equation of motion.

We will now investigate the case where the self gravity of the wall is negligible in the régimes of interest, in other words when $(G \sigma) \ll \epsilon$. Here, our equations of motion for $X$ and $h_{a b}$ remain the same, but the equation for $K_{a b}$ simplifies. Since we are neglecting gravity, the metric is flat and one can show that ${ }^{(s)} R_{a b}=K K_{a b}-K_{a}^{c} K_{c b}$. Now using equation $(4 \mathrm{a})$ and neglecting all terms proportional to $G \sigma$ we find

$$
\frac{\partial K^{a}{ }_{b}}{\partial u}=-\epsilon K^{a}{ }_{c} K^{c}{ }_{b}
$$

The solution of this equation is

$$
K_{b}^{a}=K_{b}^{a}(0)-\epsilon u K^{a}{ }_{c}(0) K_{b}^{c} .
$$

Using the trace of this equation in equation (5) we find

$$
K(0) \int_{-\infty}^{\infty} d u\left(X^{\prime}\right)^{2}+\epsilon \int_{-\infty}^{\infty} d u\left[-u\left(X^{\prime}\right)^{2} K^{a}{ }_{b}(0) K^{b}{ }_{a}+X^{\prime} D_{a} D^{a} X\right]=0
$$


We now find $K(0)$ to second order in $\epsilon$ by using an iterative procedure on equations (4b), $(6)$ and (7). Evaluating equation (7) to zeroth order in $\epsilon$ gives, as before, $K_{0}(0)=0$. It then follows from equation (6) that $K_{0}=0$. Now evaluating equation (4b) to first order and using the boundary conditions for $X$ yields $X_{1}=0$. Equation (7) to first order then gives $K_{1}(0)=0$. Now use equation (6) in equation (7) and evaluate to second order to obtain

$$
\begin{array}{cc}
K_{2}(0)\left(\int_{-\infty}^{\infty} d u X_{o}^{\prime 2}\right)+K_{o b}^{a} K_{o c}^{b} K_{o a}^{c}\left(\int_{-\infty}^{\infty} d u u^{2} X_{o}^{\prime 2}\right)=0 \\
\Rightarrow \quad K_{2}(0)=-\alpha K_{o b}^{a} K_{o c}^{b} K_{o a}^{c}
\end{array}
$$

where

$$
\alpha=\frac{\int_{-\infty}^{\infty} d u u^{2} X_{0}^{\prime 2}}{\int_{-\infty}^{\infty} d u X_{0}^{\prime 2}}>0 .
$$

Thus to second order in the wall thickness, the wall no longer obeys a Nambu equation, but acquires a term cubic in the curvature of the wall:

$$
K+\alpha \epsilon^{2} K_{0 b}^{a} K_{o c}^{b} K_{0 a}^{c}=0 .
$$

For the case of the $\lambda \phi^{4}$ wall, one finds that $\alpha=\left(\pi^{2}-6\right) / 12$ and for the Sine Gordon case $\alpha=\pi^{2} / 12$.

We now briefly remark upon walls with significant self-gravity. In this case, we have not only to worry about the behaviour of the wall surface itself, but also the way it affects the spacetime surrounding it. We therefore end up with two sets of equations which must be satisfied by the gravitating domain wall, the internal geometric equation, and the external Israel type equation. One can retain gravity in the above calculation if one wishes, and the geometric correction turns out to be similar and second order in $\epsilon$. However, the corrections to the Israel equations were worked out in [5] for the case of a $\lambda \phi^{4}$ domain wall in vacuo, and were found to be first order in the wall thickness:

$$
\left[k_{a b}\right]=-4 \pi G \tilde{\sigma} h a b,
$$

where

$$
\tilde{\sigma}=\sigma\left(1+\pi \epsilon G \sigma\left(4 \ln 2-\frac{29}{10}\right)\right)
$$


and $\left[k_{a b}\right]$ is the jump in the curvature across $\Sigma$, the surface representing the domain wall. For different models, the numerical factor in $\tilde{\sigma}$ will change, but the form of the equation remains the same. Thus, for a strongly gravitating wall, the greater effect on the wall motion is due to its self gravity. We will return to this point later.

Let us now consider the question of whether we can derive the equation of motion (10) in the absence of gravity, by an effective action argument. This would involve expanding the action

$$
S=\int \sqrt{-g}\left[\frac{1}{2}\left(\nabla_{a} \phi\right)^{2}-V(\phi)\right]
$$

in terms of the wall thickness, and integrating out perpendicular to the wall. The method would involve using the coordinate system we have already set up, and proposing an ansatz for the scalar field: $\phi=\phi_{0}(z)+\delta \phi$, where $\phi_{0}$ satisfies

$$
\frac{\partial^{2} \phi_{0}}{\partial z^{2}}-\frac{\partial V}{\partial \phi_{0}}=0
$$

and $\delta \phi$ satisfies

$$
\frac{\partial^{2} \delta \phi}{\partial z^{2}}+D_{a} D^{a} \delta \phi+K \frac{\partial \phi_{0}}{\partial z}-\frac{\partial^{2} V}{\partial \phi_{0}^{2}} \delta \phi=0
$$

The action would then be given by

$$
\begin{aligned}
S & =S\left[\phi_{0}\right]+\frac{1}{2} \int \sqrt{-g} \frac{\delta S}{\delta \phi_{0}} \delta \phi \\
& =\int \sqrt{-h}\left\{\left[-\frac{1}{2} \phi_{0}^{\prime 2}-V\left(\phi_{0}\right)\right]\left[1+\frac{1}{2} z^{2}\left(K^{2}-K_{a b}^{2}\right)\right]+\frac{1}{2} \delta \phi K \phi_{0}^{\prime}\right\} .
\end{aligned}
$$

If one used the string argument, one would argue that $\delta \phi$ would have to be proportional to $K$, and thus that the final correction term would be a $K^{2}$ term. The equation of motion for this action is derived in the appendix. Noting that the action and therefore the equation of motion is valid only to order $\epsilon^{2}$ the equation of motion to order $\epsilon^{2}$ is

$$
D_{A} D^{A} X_{2 a}\left[1+\frac{\alpha}{2} \epsilon^{2} R\right]+\alpha \epsilon^{2} D_{B} D_{A} X_{0 a} D^{B} D^{C} X_{0}^{b} D^{A} D_{C} X_{0 b}=0
$$

where $X_{0}$ satisfies

$$
D_{A} D^{A} X_{0}=0 .
$$


Thus to second order the equation of motion is

$$
D_{A} D^{A} X_{2 a}=-\alpha \epsilon^{2} D_{B} D_{A} X_{0 a} D^{B} D^{C} X_{0}^{b} D^{A} D_{C} X_{0 b}
$$

in agreement with equation (10).

At this point we want to digress momentarily to address a misconception that has arisen concerning the rigidity of cosmic strings. The effective action for strings accurate to $O\left(\epsilon^{2}\right)$ was argued to be ${ }^{6}$

$$
S=-\mu \int d^{2} \sigma \sqrt{-h}\left[1+\epsilon^{2} \Lambda\left(K_{1}^{2}+K_{2}^{2}\right)\right]
$$

where $\Lambda$ was some integral of the first order perturbations to the string fields. Using this action and the techniques of the appendix one can show that the equation of motion at zeroth order is just $K_{0}=0$ but this then implies that the first order perturbations to the fields vanish and hence $\Lambda=0$. Thus the correct equation of motion for strings accurate to order $\epsilon^{2}$ is

$$
K_{1}(0)=K_{2}(0)=0 .
$$

However, even if for some exotic type of string $\Lambda$ was not equal to zero this would still be the correct equation of motion to second order. This is because for consistency of the expansion the zeroth order solution has to be used in the equation of motion to second order obtained by varying $K^{2}$.

Thus contrary to previous claims ${ }^{6}$ to order $\epsilon^{2}$ strings are neither rigid nor antirigid. To determine their rigidity (or otherwise) the effective action to at least $O\left(\epsilon^{4}\right)$ must be obtained.

Continuing with domain walls we now apply equation (10) to a few simple cases. Firstly we consider the collapse of a cylindrical wall. Taking cylindrical coordinates $(t, \rho, \phi, z)$ for the 4-space and taking the radius $\rho$ of the cylinder at time $t$ to be $P(t)$, the normal to the wall surface is $n_{a}=\left(1-\dot{P}^{2}\right)^{-1 / 2}\left[\nabla_{a} \rho-\dot{P} \nabla_{a} t\right]$. Using equation (2) to find the extrinsic curvature of the wall we obtain

$$
K_{b}^{a}=\left(1-\dot{P}^{2}\right)^{-3 / 2}\left[\frac{1-\dot{P}^{2}}{P} \psi^{a} \psi_{b}-\ddot{P} u^{a} u_{b}\right]
$$


where $\psi_{a}$ and $u_{a}$ are given by $\psi_{a} \equiv \rho \nabla_{a} \phi$ and $u_{a}=\left(1-\dot{P}^{2}\right)^{-1 / 2}\left[\nabla_{a} t-\dot{P} \nabla_{a} \rho\right]$. Thus the equation of motion to zeroth and first order in $\epsilon$ is

$$
K(0)=\frac{\ddot{P}}{\left(1-\dot{P}^{2}\right)^{3 / 2}}+\frac{1}{P\left(1-\dot{P}^{2}\right)^{1 / 2}}=0
$$

i.e.

$$
\ddot{P}=\frac{\dot{P}^{2}-1}{P}
$$

This implies that

$$
\dot{P}^{2}=1-\left(\frac{P}{P_{i}}\right)^{2}
$$

where $P_{i}=\frac{M}{2 \pi \sigma}$ is the maximum radius of the cylinder and $M$ is its mass per unit length. This equation can be solved to obtain

$$
P=P_{i} \cos \left(\frac{t-t_{i}}{P_{i}}\right)
$$

where $t_{i}$ is the time at which $P=P_{i}$.

To second order the equation of motion is

$$
K(0)=-\epsilon^{2} \alpha K_{a}^{d} K_{e}^{a} K_{d}^{e}
$$

Using the zeroth order equations of motion it is easy to show that the correction to the equation of motion is zero. Thus (14) is true even to second order. The equation of motion is valid provided

$$
\begin{aligned}
& \left|K_{b}^{a}\right| \lesssim \epsilon \\
\Rightarrow & \frac{P}{P_{i}} \lesssim\left(\frac{\epsilon}{P_{i}}\right)^{1 / 2}
\end{aligned}
$$

as was previously suggested by Widrow ${ }^{2}$. Note that this does not agree with the naive expectation that the thin wall approximation is valid so long as the spatial radius of the cylinder is much greater than the wall's thickness. The time components of $K_{b}^{a}$ are important! 
Now consider the collapse of a spherical wall of maximum radius $R_{i}=\left(\frac{M}{4 \pi \sigma}\right)^{1 / 2}$ and mass $M$. In spherical coordinates $(t, r, \theta, \phi)$ the wall's radius is $R(t)$ and the normal to the walls surface is $n_{a}=\left(1-\dot{R}^{2}\right)^{-1 / 2}\left[\nabla_{a} r-\dot{R} \nabla_{a} t\right]$. The extrinsic curvature of the wall is given by

$$
K_{b}^{a}=\left(1-\dot{R}^{2}\right)^{-3 / 2}\left[\frac{1-\dot{R}^{2}}{R} q_{b}^{a}-\ddot{R} u^{a} u_{b}\right]
$$

where $q_{a b} \equiv r^{2}\left(\nabla_{a} \theta \nabla_{b} \theta+\sin ^{2} \theta \nabla_{a} \phi \nabla_{b} \phi\right)$ and $u_{a}=\left(1-\dot{R}^{2}\right)^{-1 / 2}\left[\nabla_{a} t-\dot{R} \nabla_{a} r\right]$.

The equation of motion to zeroth and first order in $\epsilon$ is

$$
K(0)=\frac{\ddot{R}_{0}}{\left(1-\dot{R}_{0}^{2}\right)^{3 / 2}}+\frac{2}{R_{0}\left(1-\dot{R}_{0}^{2}\right)^{1 / 2}}=0
$$

which implies that

$$
\left(1-\dot{R}_{0}^{2}\right)=\left(\frac{R}{R_{i}}\right)^{4}
$$

This equation was integrated numerically using Romberg's method of order 10 and the results is shown in figure 1. To second order in $\epsilon$ the equation of motion is

$$
\ddot{R}_{2}=-\frac{2\left(1-\dot{R}_{2}^{2}\right)}{R_{2}}+\frac{6 \epsilon^{2} \alpha}{R_{0}^{3}(t)}
$$

Note the correction term is positive and therefore finite thickness corrections lead to a slower collapse of the wall.

Equation (15) can be integrated to obtain

$$
R_{i} \dot{x}=-\sqrt{1-x^{4} e^{W\left(y^{-\theta}-1\right)}}
$$

where

$$
x=\frac{R_{2}}{R_{i}}, \quad y=\frac{R_{0}}{R_{i}}, \quad W=\frac{2 \epsilon^{2} \alpha}{R_{i}^{2}} .
$$

Figure 1 shows the result of numerically integrating (16) using a fourth order Runge-Kutta method for the case $\mathrm{W}=0.1$. The equation (16) would be expected to be a poor description 
of the collapse of the wall when $\frac{\left(W\left(y^{-6}-1\right)\right)^{2}}{2} \geq 0.1$, i.e.

$$
y \lesssim\left(\frac{W}{W+\sqrt{\frac{1}{5}}}\right)^{1 / 6}
$$

and invalid when $\left|K_{b}^{a}\right| \sim \epsilon$ i.e. when $y \sim W^{1 / 6}$ [again in agreement with Widrow ${ }^{2}$ ]. Note again that this is much sooner than naive expectations that would suggest that the approximation fails when the spatial radius of the sphere is $O(e)$.

In the case when $\frac{R_{K}}{R_{i}}>\frac{R_{i}}{\epsilon}$ where $R_{H} \sim(G \sigma)^{-1}$ the horizon size associated with the wall, the most important corrections to the equation of motion arise due to self-gravity. Using (11) [see [5] for more details] the equation of motion for the collapse of a spherical wall in the vacuum can be written to first order in $G \sigma R_{i}$ as

$$
R_{i} \dot{z}^{2}=1-\frac{z^{4}}{\left(1-S\left(1-y^{3}\right)\right)^{2}}
$$

where

$$
z=R_{1} / R_{i}, \quad S=2 \pi \sigma G R_{i}
$$

In figure 2 we show the results obtained by integrating (17) for the case $S=0.1$. Gravity can clearly be seen to reduce the rate of collapse of the wall.

Finally, it is easily checked that the exact solutions to the field theoretic equation of motion obtained by Vachaspati ${ }^{\top}$ satisfy (10) to order $\epsilon^{2}$.

To summarize, we have found the second order correction to the equation of motion of a domain wall, and exhibited that this gives a rigidity to the wall. We have shown that gravity, when important, has a stronger effect on the wall, also tending to make it more rigid. We estimated the breakdown of our approximation, which occurs typically well before the spatial radius of curvature becomes of the order of the wall thickness. Further, once this approximation does break down, corrections to all orders in thickness become important; in other words, only a field theory description will suffice to describe such a region.

For the case of cosmic strings, we argued that there was no correction to second order in the width of the string, therefore strings are Nambu to a very high accuracy, unless they 
exhibit régimes of high extrinsic curvature (such as cusps). At such places, oniy a field theoretic description will suffice. However, note that this breakdown will occur well before the spatial radius of curvature becomes of order the string width.

Finally, Nielsen and Olesen were interested in a vortex solution exhibiting Nambu properties in order to get a phenomenological representation of a dual string. The lack of the second order correction term in the case of strings shows that the " $\sqrt{\gamma}\left(1+k^{2}\right)$ " lagrangian first suggested by Polyakov ${ }^{8}$, has no physical manifestation.

\section{Appendix.}

Here we will find the equations of motion associated with the action (11). In order to do this, we use an alternate definition of the curvature in terms of the coordinates of $\Sigma$, which we will denote as $X^{a}\left(\xi^{A}\right), \xi^{A}$ being the intrinsic coordinates of the surface $\Sigma$ :

$$
D_{A} D_{B} X^{a}=K_{A B} n^{a}
$$

We therefore need to find the equations of motion associated with the action

$$
S=-\sigma \int d^{3} \xi \sqrt{-h}\left[1+\beta \epsilon^{2}\left(D_{A} D^{A} X^{a}\right)^{2}-\Delta \epsilon^{2} D_{A} D_{B} X^{a} D^{A} D^{B} X_{a}\right]
$$

where $\Delta=\alpha / 2$. In varying the action with respect to $X^{a}$, we must remember that both the metric, $h_{A B}$, and the connection, $h_{A B}^{C}$ depend on $X^{a}$. For the metric we have:

$$
\delta h_{A B}=\delta X_{, A}^{a} X_{a, B}+X_{, A}^{a} \delta X_{a, B}
$$

Therefore, varying $S$ with respect to $X^{a}$ gives three pieces The first term is the variation of the volume element, this gives, upon integration by parts,

$$
\delta S_{1}=\sigma \int d^{3} \xi \sqrt{-h} \delta X^{a} D_{A}\left[D^{A} X_{a}\left[1+\beta \epsilon^{2}\left(D_{C} D^{C} X^{b}\right)^{2}-\Delta \epsilon^{2}\left(D_{B} D_{C} X^{a}\right)^{2}\right]\right]
$$

The other two terms contain the variation

$$
\delta\left[h^{A C} D_{A} D_{B} X^{a}\right]=-h^{A E} h^{C D} \delta h_{E D} D_{A} D_{B} X^{a}+D^{C} D_{B} \delta X^{a}-h^{A C} \delta h_{A B}^{E} X^{a}{ }_{E}
$$

the last term of which vanishes when contracted with $D D X_{a}$, which is normal to the wall. 
Substituting from (A3) and (A5) and integrating by parts as necessary gives

$$
\delta S_{2}=\sigma \beta \epsilon^{2} \int d^{3} \xi \sqrt{-h}\left\{4 D_{C}\left[D_{B} D^{B} X_{b} D_{A} D^{C} X^{b} D^{A} X_{a}\right]+2\left(D_{A} D^{A}\right)^{2} X_{a}\right\} \delta X^{a},
$$

and

$$
\delta S_{3}=-\sigma \Delta \epsilon^{2} \int d^{3} \xi \sqrt{-h}\left\{4 D_{C}\left[D^{C} D^{B} X_{b} D_{A} D_{B} X^{b} D^{A} X_{a}\right]+2 D_{B} D_{A} D^{A} D^{B} X_{a}\right\} \delta X^{a}
$$

Now, the Gauss Codazzi equations imply

$$
R_{A C}=K K_{A C}-K_{A B} K_{C}^{B}=D_{B} D^{B} X_{b} D_{A} D_{C} X^{b}-D_{A} D_{B} X_{b} D^{B} D_{C} X^{b}
$$

and hence

$$
\begin{aligned}
\delta S_{3} & =-\sigma \Delta \epsilon^{2} \int d^{3} \xi \sqrt{-h}\left\{4 D_{C}\left[D_{B} D^{B} X_{b} D_{A} D^{C} X^{b} D^{A} X_{a}\right]-2 D_{B}\left[R^{A B} D_{A} X_{a}\right]\right. \\
& \left.+2 D_{A} D^{A} D_{B} D^{B} X_{a}\right\} \delta X^{a} .
\end{aligned}
$$

Thus, imposing $\frac{\delta S}{\delta X^{\circ}}=0,(\mathrm{~A} 4),(\mathrm{A} 6)$ and (A9) give as the equations of motion for the wall:

$$
\begin{aligned}
& D_{A}\left[D^{A} X_{a}\left[1+\beta \epsilon^{2}\left(D_{C} D^{C} X^{b}\right)^{2}-\Delta \epsilon^{2}\left(D_{B} D_{C} X^{a}\right)^{2}\right]\right]+2(\beta-\Delta) \epsilon^{2}\left(D_{A} D^{A}\right)^{2} X_{a} \\
& +4(\beta-\Delta) \epsilon^{2} D_{C}\left[D_{B} D^{B} X_{b} D_{A} D^{C} X^{b} D^{A} X_{a}\right]-2 \Delta \epsilon^{2} D_{B}\left[R^{A B} D_{A} X_{a}\right]=0 .
\end{aligned}
$$

Now, from our previous work, we know that the first order corrections vanish, and hence $\beta=\Delta$. This can also be deduced if we use the zeroth order form of the above equation $\left(D_{A} D^{A} X^{a}=0\right)$ and input this information back into the argument at the stage of 'solving' for the field perturbations. (One may regard this as slightly circular.) However, using this simplification one obtains

$$
D_{A} D^{A} X_{a}\left[1+\Delta \epsilon^{2} R\right]+2 \Delta \epsilon^{2} D_{B} D_{A} X_{a} D^{B} D^{C} X^{b} D^{A} D_{C} X_{b}=0
$$

Which is the equation of motion we wished to derive.

\section{Acknowledgements.}

We would like to thank Michael Turner for interesting discussions. This work was supported in part by the U.S. Department of Energy and NASA grant NAGW-1340 at Fermilab, and by NSF grant PHY-85-06686 to the University of California. 


\section{Figure Captions}

(1)The collapse of a spherically symmetric domain wall to zeroth order (solid line) and second order (long dashed line). The horizontal dashed line corresponds to $y=$ $\left(\frac{W}{W+\sqrt{1 / 5}}\right)^{1 / 6}$

(2)The collapse of a spherically symmetric domain wall for the case $S=0.1$ to zeroth order (solid line) and second order (dashed line)

\section{References}

[1] C.Hill, J.Fry, D.Schramm, Phys. Rev. D39 3571 (1989).

A.Stebbins, M.Turner, FERMILAB-PUB-88/170-A

[2] L.Widrow, Phys. Rev. D39 3571 (1989). and Phys. Rev. D40 1002 (1989).

[3] W.Press, B.Ryden, D.Spergel, NSF-ITP-89-51

[4] This has independently been noted by J.Simon, UCSB-89-50

[5] D.Garfinkle, R.Gregory, FERMILAB-Pub-89/255-A

[6] K.Maeda, N.Turok, Phys. Lett. 3B 376 (1988).

R.Gregory, Phys. Lett. 199B 206 (1988).

[7] T.Vachaspati, Vachaspati, TUTP 89-7

[8] A. Polyakov, Nucl. Phys. B268 406 (1986). 


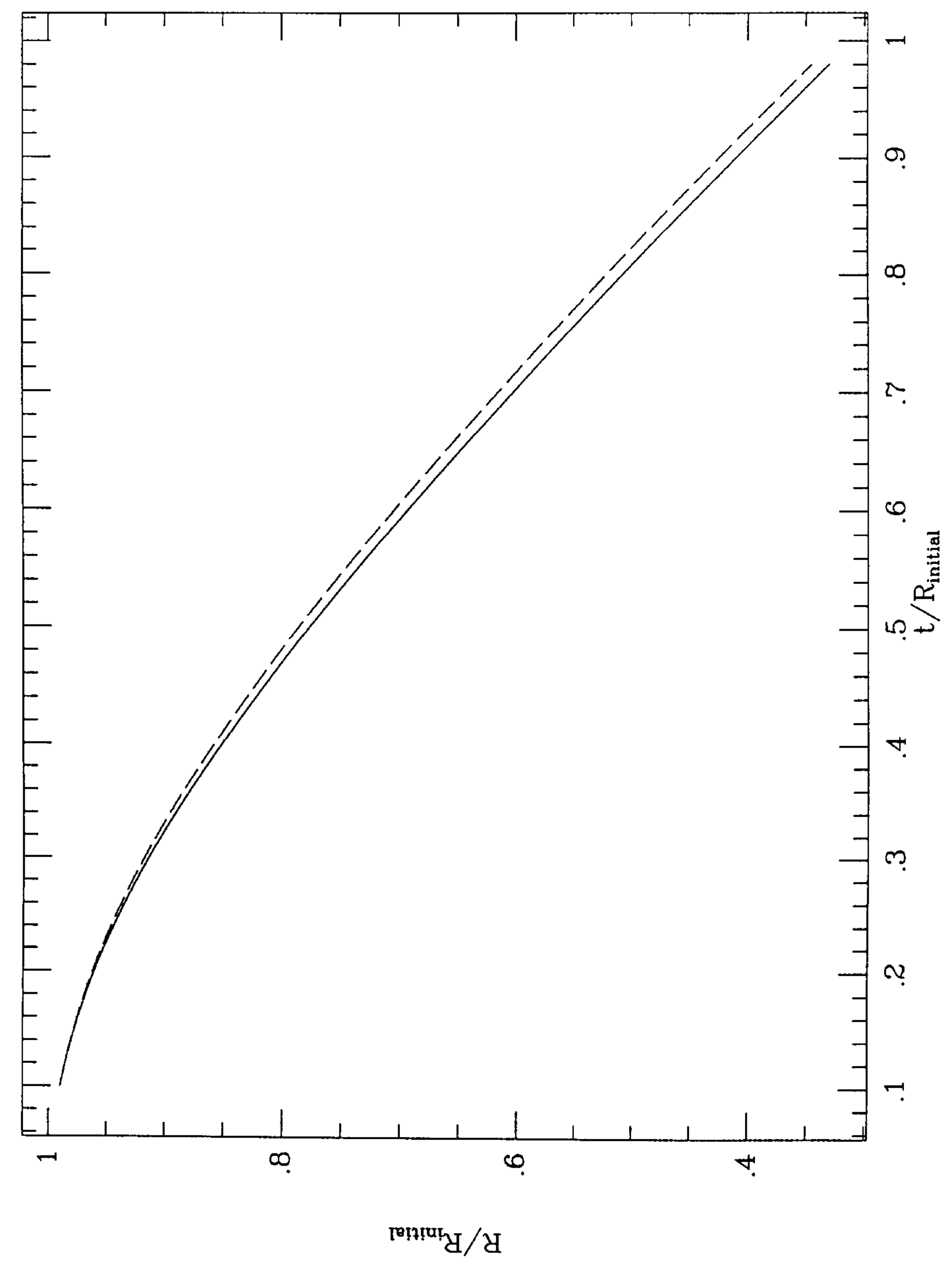




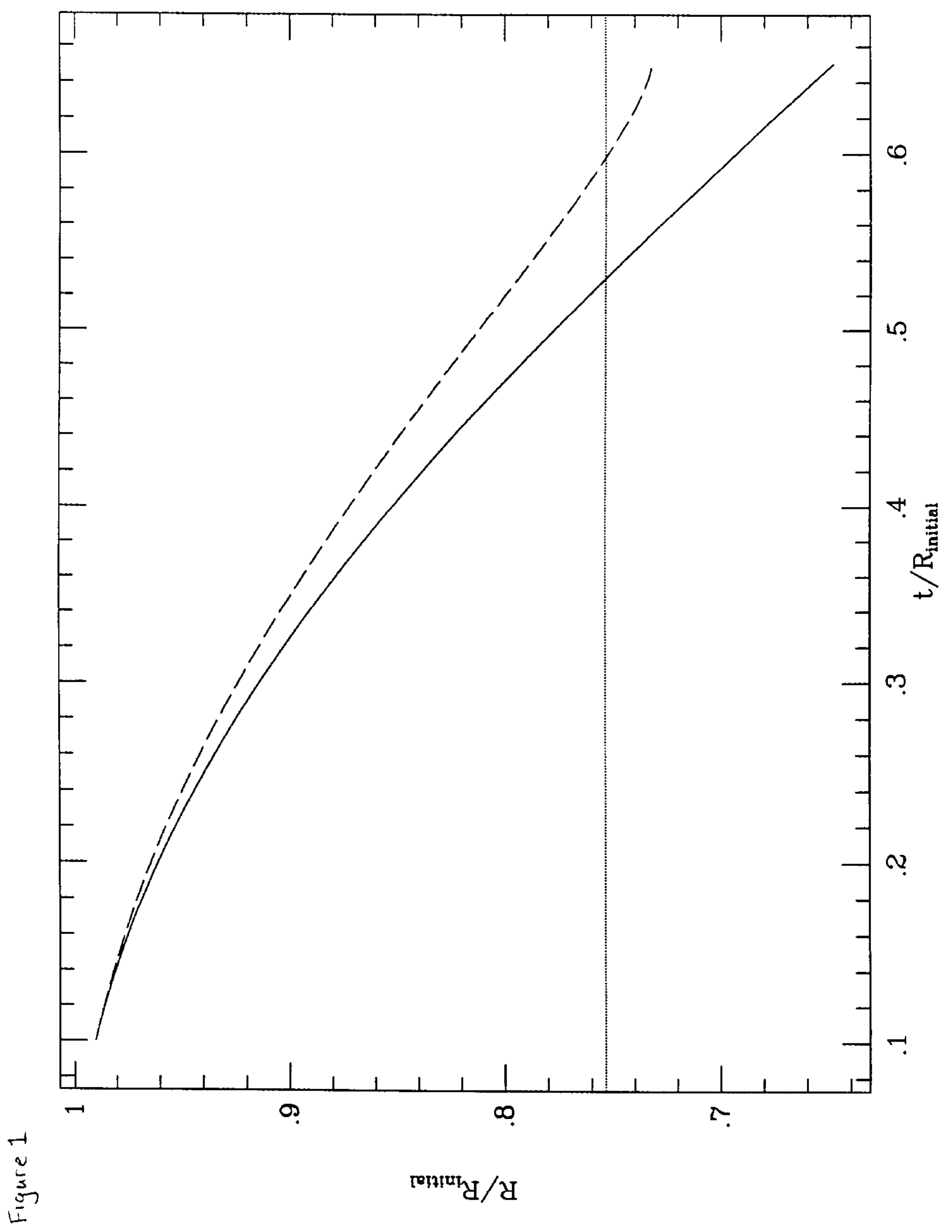

